\title{
Croissance radiale du pin d'Alep (Pinus halepensis Mill.) en fonction des paramètres stationnels dans les massifs forestiers naturels de l'Atlas saharien algérien
}

Brahim GuIT ${ }^{1}$

Bouzid NedJIMI ${ }^{1}$

${ }^{1}$ Université Ziane Achour

Faculté des Sciences de la Nature et de la Vie

Laboratoire d'exploration et de valorisation des écosystèmes steppiques Cité Aïn Chih, BP 3117

Djelfa 17000

Algérie

Auteur correspondant / Corresponding author:

Bouzid NEDJIMI - bnedjimi@yahoo.fr

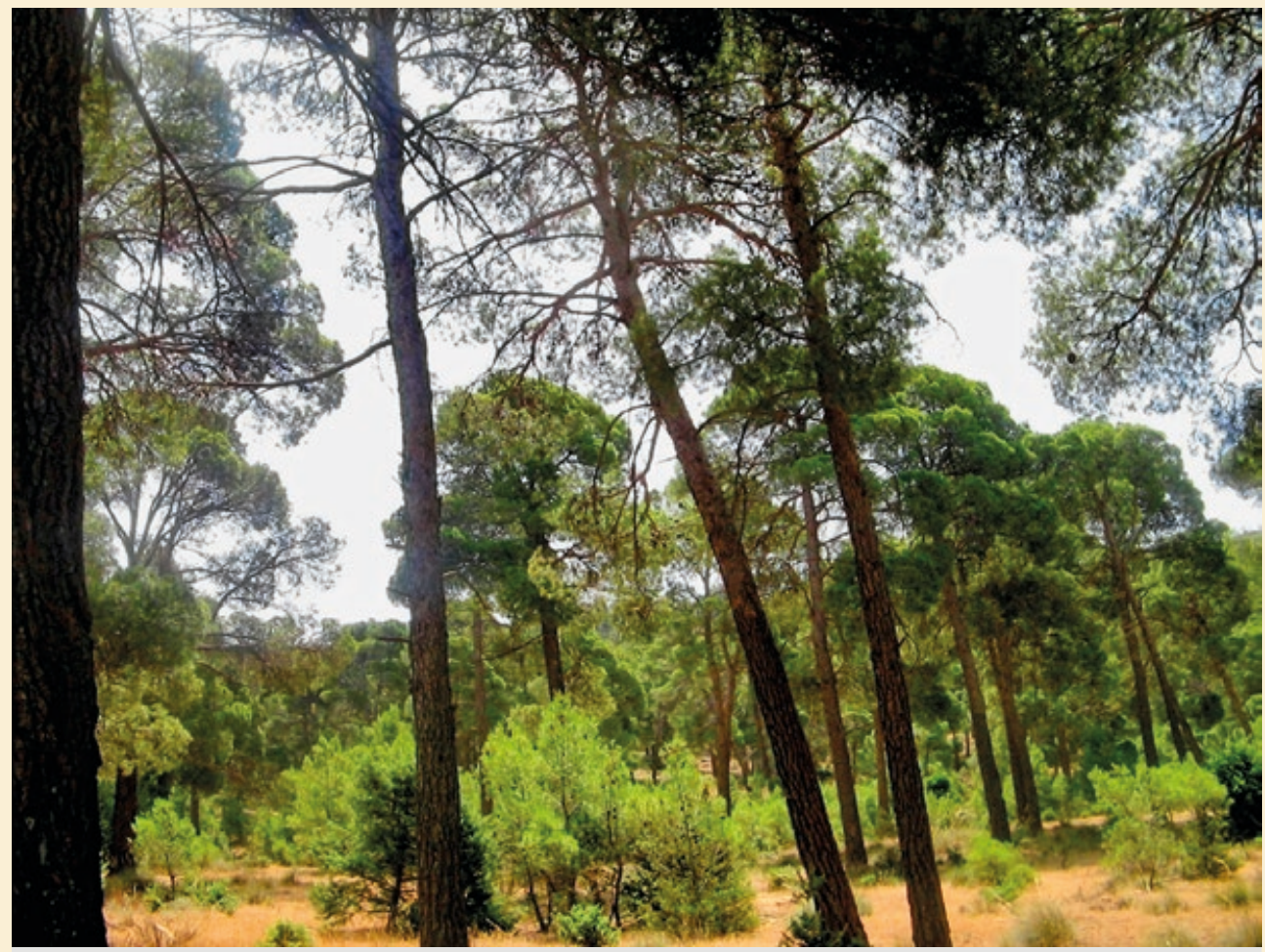

Site d'échantillonnage de la station de Senalba Chergui. Photo B. Guit.

Doi : 10.19182/bft2020.345.a31803 - Droit d'auteur (c) 2020, Bois et Forêts des Tropiques - (c) Cirad - Date de soumission : 19 novembre 2019 ; date d'acceptation : 11 mai 2020 ; date de publication : $1^{\text {er }}$ novembre 2020.

\section{ecirad a)}

(ब) (1)
Licence Creative Commons :

Attribution - 4.0 International.

Attribution-4.0 International (CC BY 4.0)
Citer l'article / To cite the article

Guit B., Nedjimi B., 2020. Croissance radiale du pin d'Alep (Pinus halepensis Mill.) en fonction des paramètres stationnels dans les massifs forestiers naturels de l'Atlas saharien algérien. Bois et Forêts des Tropiques, 345 : 3-11. Doi : https://doi.org/10.19182/bft2020.345.a31803 


\section{RÉSUMÉ}

\author{
Croissance radiale du pin d'Alep (Pinus \\ halepensis Mill.) en fonction des \\ paramètres stationnels dans les massifs \\ forestiers naturels de l'Atlas saharien \\ algérien
}

La présente étude dendroécologique a été conduite dans les pinèdes de Senalba Gharbi, Senalba Chergui et Sehary Guebli situées en zone semi-aride de la région de Djelfa (Atlas saharien algérien) et caractérisées par une pluviométrie variant entre 300 et $400 \mathrm{~mm} / \mathrm{an}$. Ce sont les derniers massifs arborescents, peuplés de pin d'Alep, Pinus halepensis, situés à la limite du Sahara. Le maintien de ces peuplements soumis à une dégradation continuelle depuis plusieurs décennies constitue un enjeu écologique majeur. À partir de transects intégrant les variations d'altitude, d'exposition et de position topographique, 18 placettes d'échantillonnage ont été sélectionnées. Dans chaque placette, six arbres parmi les plus dominants ont été sondés à la tarière de Pressler, à raison de deux carottes de sondage par arbre, soit un total de 216 carottes-échantillons analysées sur une période de 53 ans. L'analyse des cernes a mis en évidence que l'exposition, l'altitude et la position topographique sont déterminantes pour l'accroissement des pins. L'étude des séries dendrochronologiques relatives à la largeur des cernes a montré une tendance générale décroissante de $10,5 \%$. La prise en compte de ces données relatives à la croissance radiale contribuera à identifier les arbres à forte probabilité de déclin suite aux changements climatiques.

Mots-clés : Pinus halepensis, forêt semi-aride, dendroécologie, position topographique, changement climatique, Algérie.

\section{ABSTRACT}

\author{
Radial growth of Aleppo pine (Pinus \\ halepensis Mill.) according to biotope \\ parameters for the natural forests \\ of Algeria's Saharan Atlas
}

This study was made in the forests of Senalba Gharbi, Senalba Chergui and Sehary Guebli, in the semi-arid zone of Djelfa (Algerian Saharan Atlas), which is characterized by rainfall of 300 to $400 \mathrm{~mm} /$ year. These are the last tree stands with Aleppo pine, Pinus halepensis, growing at the edge of the Sahara. To investigate the effects of altitude, exposure and topographic position on tree measurements (radial growth), 18 plots were selected for sampling over a period of 53 years. In each plot, two samples were taken from six of the dominant trees, using an increment borer, making 216 samples altogether. Analysis of the tree rings showed that exposure, altitude and topographic position have a significant effect on the width of the pine tree rings. Analysis of the dendrochronological series of tree-ring sizes then showed an overall decreasing trend of $10.5 \%$. Radial growth data can thus be usefully analysed to identify trees with a high probability of decline due to climate change.

Keywords: Pinus halepensis, semi-arid forests, dendroecology, topographic position, climate change. Algeria.

\section{RESUMEN}

\author{
Crecimiento radial de un pino de Alepo \\ (Pinus halepensis Mill.) en función \\ de los parámetros estacionales \\ en los macizos forestales del Atlas \\ sahariano argelino
}

El presente estudio dendroecológico fue llevado a cabo en las pinedas de Senalba Gharbi, Senalba Chergui y Sehary Guebli, situadas en zona semiárida de la región de Djelfa (Atlas sahariano argelino) y caracterizadas por una pluviometría que varía entre 300 y $400 \mathrm{~mm} / a$ ño. Se trata de los últimos macizos arborescentes poblados con pino de Alepo, Pinus halepensis, situados en el límite del Sahara. El mantenimiento de esta masa forestal, sometida a una degradación continua desde hace varios decenios, constituye un reto ecológico importante. A partir de transectos que integran las variaciones de altitud, de exposición y de posiciones topográficas, se seleccionaron 18 parcelas de muestreo. En cada parcela de ensayo, se sondaron seis árboles de entre los más dominantes con la barrena de Pressler, con dos bastoncillos de extracción por árbol, es decir, un total de 216 muestras analizadas en un período de 53 años. El análisis de los anillos de crecimiento evidenció que la exposición, la altitud y la posición topográfica son determinantes para el crecimiento de los pinos. El estudio de las series dendrocronológicas relativas al espesor de los anillos mostró una tendencia general decreciente del 10,5 \%. Estos datos relativos al crecimiento radial podrían utilizarse para identificar los árboles con alta probabilidad de declive debido a los cambios climáticos.

Palabras clave: Pinus halepensis, bosque semiárido, dendroecología, posición topográfica, cambio climático, Argelia. 


\section{Introduction}

Les changements climatiques qui ont pris de l'ampleur durant ces dernières décennies se traduisent par une augmentation de la fréquence, de la durée et/ou de l'intensité des épisodes de sécheresse et de stress thermique. Ils ont pour conséquences des changements fondamentaux de la composition, de la structure et de la biogéographie des forêts dans de nombreuses régions du monde (Allen et al., 2010). La région méditerranéenne est une zone où ces changements sont marqués car cette région constitue une zone de transition entre le climat aride du nord de l'Afrique et celui de l'Europe tempérée et pluvieuse (Ruiz-Labourdette et al., 2013). Ces dérèglements climatiques ont un impact direct sur la croissance, la productivité et la distribution actuelle de la végétation méditerranéenne (De Dios et al., 2007 ; IPCC, 2007). Les écosystèmes forestiers sont particulièrement sensibles à ces modifications car la longue durée de vie des arbres ne permet pas une adaptation rapide à ces changements environnementaux (Lindner et al., 2010).

L'analyse des cernes ligneux est de plus en plus utilisée pour comprendre les changements environnementaux et écologiques. Elle fournit en effet des informations à haute résolution sur la croissance radiale des arbres sur de longues périodes, à travers de vastes territoires et avec des gradients environnementaux (Babst et al., 2013 ; D’Orangeville et al., 2016; Girardin et al., 2016). Les caractéristiques des cernes permettent de quantifier la sensibilité des arbres aux variations de climat à long terme (Fritts, 2001).

De Luis et al. (2013) considèrent que le pin d'Alep est une espèce cible de référence pour les études dendroclimatologiques. Cette essence est la plus répandue dans le bassin méditerranéen occidental où elle domine dans les endroits les plus secs et les plus chauds, couvrant ainsi environ 3,5 millions d'hectares de peuplements naturels et de reboisements (Ne'eman et Trabaud, 2000). En Algérie, l'espèce couvre $35 \%$ des surfaces boisées du Nord, soit environ 800000 ha (Bentouati et al., 2005). Elle est localisée en grande partie à l'état naturel dans les régions de l'Est et du Centre du pays, principalement sur les Atlas tellien et saharien. Les principales forêts sont réparties sur les crêtes de l'Atlas saharien entre 1000 et 1500 m d'altitude (Kadik, 1987).

La présente étude porte sur une étude dendroécologique du pin d'Alep en fonction des paramètres stationnels (exposition, altitude et position topographique) dans les massifs forestiers de Senalba Gharbi, Senalba Chergui et Sehary Guebli, qui constituent une des plus importantes forêts naturelles de pin d'Alep de l'Atlas saharien algérien.

\section{Matériel et méthodes}

\section{Site d'étude}

Notre étude a été conduite dans les massifs forestiers de Senalba Gharbi, Senalba Chergui et Sehary Guebli situés dans les Monts des Ouled Naïl. Ces massifs sont localisés en grande partie sur les collines des hauts plateaux de l'Atlas saharien à environ 300 kilomètres au sud d'Alger (figure 1). Les forêts domaniales de Senalba Chergui, de Senalba Gharbi et de Sehary Guebli se situent respectivement à 4 km à l'ouest, à $25 \mathrm{~km}$ au sud-ouest et à $20 \mathrm{~km}$ au nord-est de la commune de Djelfa.

Ces massifs occupent trois crêtes orientées sud-ouest / nord-est et s'étendent sur plus de $60 \mathrm{~km}$. Ces trois crêtes descendent en pente douce au sud, mais en pente abrupte au nord vers les hautes plaines. Les pentes sont comprises entre environ $40 \%$ vers les sommets et 5 à $20 \%$ au bas des versants. L'altitude de ces massifs varie de 1100 à 1530 m.

La zone d'étude est située en bioclimat semi-aride à hiver froid. La pluviométrie annuelle moyenne est de $319 \pm 77 \mathrm{~mm}$ pour la période 1984-2013. La température moyenne annuelle est de $14,11 \pm 0,5^{\circ} \mathrm{C}$. Le mois de janvier est le mois le plus froid avec une température minimale moyenne de $4,4^{\circ} \mathrm{C}$. La température du mois le plus chaud (juillet) est d'environ $34^{\circ} \mathrm{C}$.

Les sols sont caractérisés par une hétérogénéité liée à la roche-mère et à la topographie. Kadik $(1987,2005)$ distingue deux classes de sols : les rendzines et les sols bruns calcaires. La profondeur du sol est de $40 \mathrm{~cm}$ en moyenne et diminue depuis le bas vers le haut du versant.

On distingue trois strates de végétation : une strate arborescente constituée exclusivement de pin d'Alep; une strate arbustive avec de nombreux arbustes parmi lesquels le chêne vert (Quercus ilex), le genévrier de Phénicie (Juniperus phoenicea), le genévrier oxycèdre (Juniperus oxycedrus), le filaire (Phillyrea angustifolia) et les pistachiers (Pistacia lentiscus et Pistacia terebinthus) ; une strate herbacée dominée par deux graminées, le diss (Ampelodesma mauritanicum) et l'alfa (Stipa tenacissima).

La densité moyenne des arbres au sein des trois massifs est de 256 pieds par hectare. Elle varie en fonction de l'exposition, de l'altitude, de l'activité humaine et des traitements sylvicoles appliqués. Deux aménagements forestiers ont été réalisés. Le premier, mené par un bureau d'études bulgare en 1984, a été suivi d'une révision d'aménagement en 1994 par le Bureau national des études forestières. Les éclaircies ont touché pour la plupart les pins dont le diamètre dépassait $35 \mathrm{~cm}$, soit les classes de circonférences supérieures à $100 \mathrm{~cm}$, et dont l'âge dépassait largement l'âge d'exploitabilité.

Les sites échantillonnés correspondent à une distribution assez étendue du pin d’Alep dans la région semi-aride centre. Trois critères de choix ont été adoptés :

- l'altitude, selon un transect altitudinal du bas vers le haut du versant, trois positions géomorphologiques ayant été retenues, bas versant, mi-versant et haut versant ; 


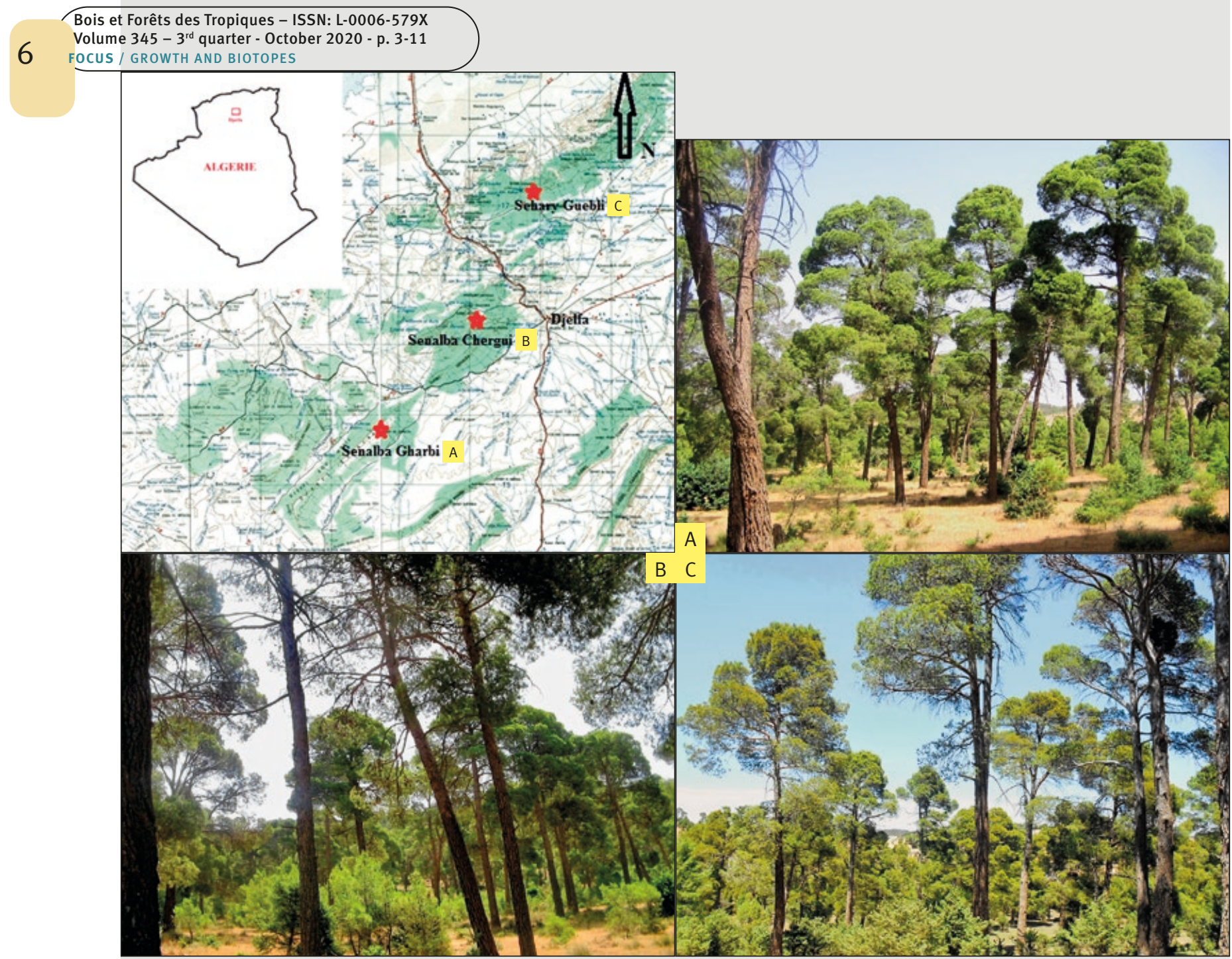

Figure 1.

Carte de situation des sites d'échantillonnage : station de Senalba Gharbi (A),

station de Senalba Chergui (B), station de Sehary Guebli (C).

Photos B. Guit.

- l'exposition, nord et sud pour chacun des trois massifs forestiers ;

- l'âge des peuplements, sachant qu'il s'agit d'une vieille futaie de pin d'Alep dont les arbres ont en moyenne 100 ans (figure 1) ; les individus les plus sénescents atteignent 150 ans alors que les plus jeunes ont 60 ans. L'analyse dendrochronologique n'a concerné que les cernes formés après 1960 afin de s'affranchir de l'effet « juvénilité ».

Le tableau I résume les caractéristiques des sites échantillonnés.

\section{Sélection des arbres étudiés et mesures réalisées}

La croissance des arbres a été étudiée sur des carottes de sondage prélevées à l'aide d'une tarière de Pressler dans les arbres échantillonnés.

La sélection des arbres ainsi sondés a pris en compte les individus dominants sur chaque site. En dendroécologie, les arbres dominants sont privilégiés dans les stratégies d'échantillonnage car leur croissance est moins affectée par la compétition inter-individus que les arbres de petits diamètres, souvent dominés (Lebourgeois et Mérian, 2012). Au total, six arbres parmi les plus dominants ont été sondés au niveau de chacune des six positions topographiques, c'est-à-dire les trois positions géomorphologiques croisées avec les deux expositions nord et sud. Le nombre total de pins sondés est ainsi de 108 individus. Les circonférences moyennes des arbres sondés correspondants sur chaque massif sont les suivantes : Senalba Gharbi : $110 \pm 25 \mathrm{~cm}$; Sehary Guebli : $95 \pm 21 \mathrm{~cm}$; Senalba Chergui : $84 \pm 19 \mathrm{~cm}$.

Deux carottes ont été prélevées dans chaque arbre suivant les directions nord-sud. Les carottes extraites ont été séchées à l'air puis poncées et interdatées (Fritts et Swetnam, 1989). Les épaisseurs des cernes ont été mesurées à l'aide d'un appareil de mesure LINTAB 6, qui a fait des mesures à 0,01 mm, couplé au logiciel TSAP $®$.

Les épaisseurs de cernes sur chaque carotte correspondent à une série élémentaire ; la moyenne de deux séries élémentaires des deux carottes d'un même arbre correspond à une série individuelle pour chaque arbre. 
Tableau I.

Caractéristiques des sites échantillonnés.

\begin{tabular}{|c|c|c|c|c|c|c|}
\hline Site & Latitude & Longitude & $\begin{array}{l}\text { Altitude } \\
\text { moyenne }\end{array}$ & $\begin{array}{c}\text { Profondeur moyenne } \\
\text { du sol }(\mathrm{cm})\end{array}$ & Âge moyen & $\begin{array}{l}\text { Densité moyenne } \\
\text { (pieds/ha) }\end{array}$ \\
\hline Sehary Guebli & $34^{\circ} 50^{\prime} 23^{\prime \prime} \mathrm{N}$ & $3^{\circ} 13^{\prime} 58^{\prime \prime} \mathrm{E}$ & 1104 m & $46,7 \pm 7,6$ & $103 \pm 21$ & $280 \pm 61$ \\
\hline Senalba Chergui & $34^{\circ} 38^{\prime} 37^{\prime \prime} \mathrm{N}$ & $3^{\circ} 09^{\prime} 31^{\prime \prime} \mathrm{E}$ & $1210 \mathrm{~m}$ & $42,7 \pm 8,9$ & $101 \pm 20$ & $334 \pm 110$ \\
\hline Senalba Gharbi & $34^{\circ} 31^{\prime} 21^{\prime \prime} \mathrm{N}$ & $2^{\circ} 58^{\prime} 39^{\prime \prime} \mathrm{E}$ & 1323 m & $30,1 \pm 8,9$ & $125 \pm 22$ & $154 \pm 42$ \\
\hline
\end{tabular}

Tableau II.

Moyenne des largeurs des cernes (en $\mathrm{mm}$ ) par massif et par position topographique.

\begin{tabular}{|c|c|c|c|c|}
\hline Exposition & Position topographique & Senalba Gharbi & Senalba Chergui & Sehary Guebli \\
\hline \multirow[t]{4}{*}{ Nord } & Bas versant & $1,34 \pm 0,22$ & $1,25 \pm 0,25$ & $1,21 \pm 0,16$ \\
\hline & Mi-versant & $1,25 \pm 0,13$ & $1,19 \pm 0,17$ & $1,18 \pm 0,18$ \\
\hline & Haut versant & $1,21 \pm 0,14$ & $1,12 \pm 0,17$ & $1,10 \pm 0,28$ \\
\hline & Moyenne du versant & $1,26 \pm 0,17$ & $1,18 \pm 0,19$ & $1,16 \pm 0,20$ \\
\hline \multirow[t]{4}{*}{ Sud } & Bas versant & $1,22 \pm 0,32$ & $1,21 \pm 0,23$ & $1,14 \pm 0,27$ \\
\hline & Mi-versant & $1,27 \pm 0,46$ & $1,18 \pm 0,32$ & $1,12 \pm 0,16$ \\
\hline & Haut versant & $1,18 \pm 0,31$ & $1,10 \pm 0,15$ & $1,08 \pm 0,31$ \\
\hline & Moyenne du versant & $1,22 \pm 0,36$ & $1,16 \pm 0,24$ & $1,11 \pm 0,25$ \\
\hline Moyenne du massif & & $1,24 \pm 0,26$ & $1,17 \pm 0,21$ & $1,13 \pm 0,22$ \\
\hline
\end{tabular}

\section{Résultats et discussion}

La chronologie moyenne de chaque position topographique résulte de la moyenne, année par année, des épaisseurs de cernes de toutes les séries élémentaires. La chronologie maîtresse de chaque massif est la moyenne des chronologies de toutes les positions au niveau des deux expositions.

L'épaisseur moyenne des cernes est égale à la somme de toutes les épaisseurs des cernes d'une chronologie divisée par le nombre de cernes.

Les années caractéristiques correspondent aux dates pour lesquelles au moins $75 \%$ des arbres du peuplement considéré présentent une variation relative de croissance d'au moins $10 \%$ par rapport à l'année précédente. Nous considérons comme années exceptionnelles celles pour lesquelles le total des précipitations moyennes est inférieur ou supérieur à la moyenne de la série climatique, diminuée de la valeur de l'écart-type (Safar, 1994).

Une analyse de variance à un facteur a été réalisée pour tester l'influence de l'exposition, de l'altitude et de la position topographique sur la largeur du cerne. 


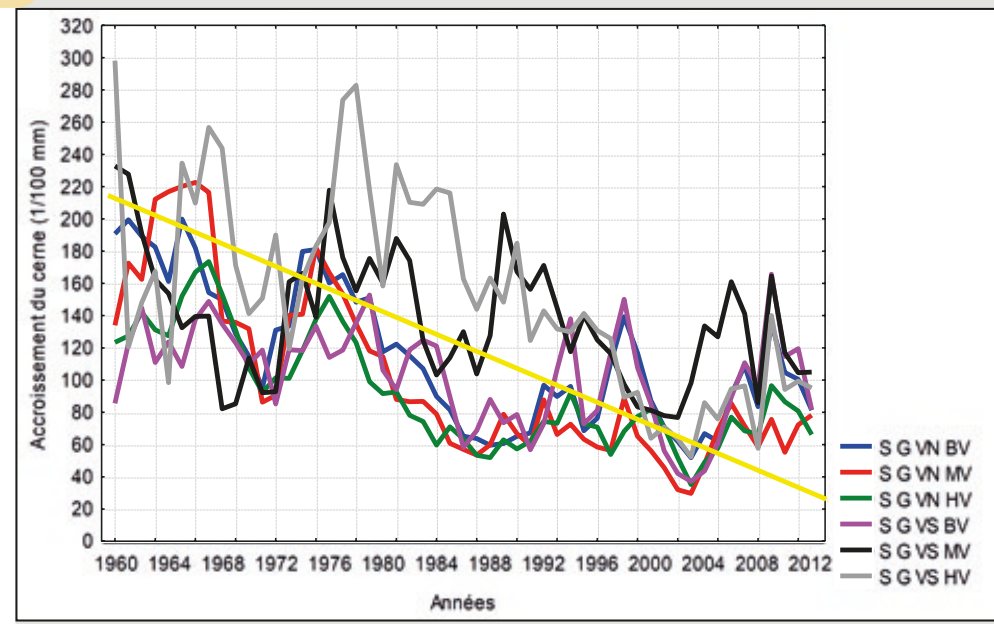

Figure 2.

Séries chronologiques, massif de Senalba Gharbi.

S G : Senalba Gharbi ; VN : versant nord ; VS : versant sud ; $\mathrm{BV}$ : bas versant ; $\mathrm{MV}$ : mi-versant ; $\mathrm{HV}$ : haut versant.

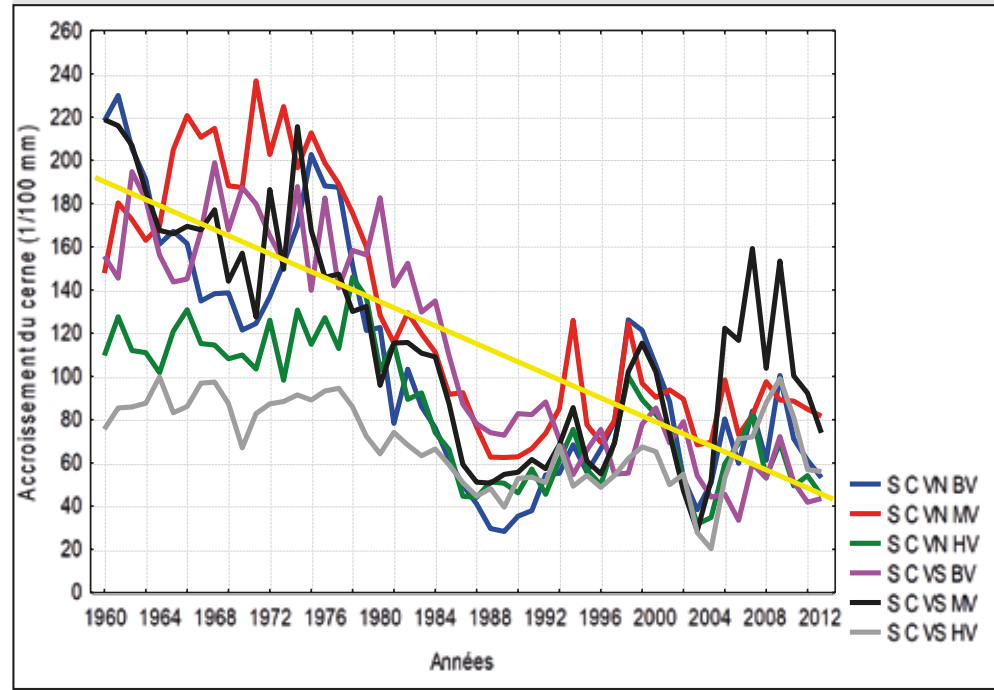

Figure 3.

Séries chronologiques, massif de Senalba Chergui.

S C : Senalba Chergui ; VN : versant nord ; VS : versant sud ;

$\mathrm{BV}$ : bas versant ; MV : mi-versant ; HV : haut versant.

de Senalba Gharbi et Senalba Chergui ; en versant sud, ces valeurs sont respectivement de 1,20 et $1,10 \mathrm{~mm}$ pour les deux forêts. Ils notent ainsi une meilleure croissance en versant nord qu'en versant sud de ces deux massifs, résultat identique à celui de la présente étude.

Dans une étude dendroécologique du pin d'Alep dans dans l'Atlas saharien, sur une période de 75 ans (19141988), Safar (1994) a obtenu des épaisseurs de cernes de $1,47 \mathrm{~mm}$ et $1,46 \mathrm{~mm}$ pour la même région de Senalba.

Concernant l'influence de l'exposition sur la croissance du pin d'Alep, Carmel et Kadmon (1999) et Osem et al. (2009) soulignent qu'il est classiquement admis que la plus faible intensité du rayonnement solaire sur les versants nord associée à une moindre sécheresse favorise la croissance et la productivité des forêts méditerranéennes arides. Schiller (1972), dans son étude sur les facteurs écologiques affec- tant la croissance du pin d'Alep dans la colline de Judée du Sud (Israël), montre que l'exposition des versants n'a qu'une faible influence sur les performances des arbres dans la forêt de Sha'ar Ha'Gay (632 mm de précipitations annuelles moyennes), alors qu'à Lahav et Yatir, correspondant à la partie sud des collines de Judée $(300 \mathrm{~mm}$ de précipitations annuelles moyennes), l'exposition a une influence considérable sur la croissance des arbres.

La comparaison des épaisseurs de cernes entre les trois massifs montre que le pin d'Alep présente un meilleur accroissement dans la forêt de Senalba Gharbi par rapport aux deux autres forêts. L'étude de la variance a révélé un effet significatif ( $p$-value $=0,041$ ) du facteur " altitude " sur les épaisseurs de cernes qui sont respectivement de $1,24 \mathrm{~mm}, 1,17 \mathrm{~mm}$ et $1,13 \mathrm{~mm}$ pour Senalba Gharbi, Senalba Chergui et Sehary Guebli.

Kaabèche (1996) note que les forêts de pin d'Alep de l'Atlas saharien algérien se trouvent en limite méridionale absolue. Aussi, elles recherchent des compensations "mésoclimatiques " liées à l'altitude (partie supérieure des massifs) et à l'exposition (versant nord).

La position topographique s'avère déterminante pour l'accroissement du pin d'Alep ; l'analyse de la variance a mis en évidence un effet très hautement significatif de ce facteur sur l'épaisseur des cernes ( $p$-value $<0,0001$ ).

En moyenne, pour les trois forêts et les deux expositions, la largeur des cernes est de $1,22 \mathrm{~mm}$ en bas de versant contre $1,19 \mathrm{~mm}$ à mi-versant et $1,13 \mathrm{~mm}$ en haut de versant.

La profondeur du sol peut avoir un impact sur ce résultat puisqu'elle croît avec la position topographique, avec respectivement $46,6 \pm 9,5 \mathrm{~cm}, 38,4 \pm 8,5 \mathrm{~cm}$ et $32,2 \pm 8,1 \mathrm{~cm}$ en haut de versant, à mi-versant et en bas de versant.

Ces résultats concordent avec ceux de Brochiero et al. (1999) qui, dans leur étude traitant de l'autécologie et de la croissance du pin d'Alep en Provence calcaire (France), mentionnent que la topographie générale est déterminante pour la croissance du pin d'Alep. Dans les vallées, les vallons, les plaines et sur les plateaux, le pin d'Alep présente une meilleure croissance par rapport aux hauts de pente qui sont beaucoup moins favorables. Ils concluent qu'un bilan hydrique élevé est synonyme de bonne fertilité.

\section{Caractéristiques dendrochronologiques}

Les figures 2 à 5 présentent les chronologies d'épaisseurs de cernes des trois positions topographiques pour les trois massifs ainsi que la tendance globale de 1960 jusqu'à 2012 (soit une période de 53 ans).

L'année 1960 a été fixée comme point de départ des séries chronologiques car le cœur de certains arbres n'a pas pu être atteint lors du sondage. En outre, sur certains arbres à gros diamètre, la tarière de Pressler utilisée n'était pas suffisamment longue pour en atteindre le cœur.

Le tableau III présente les droites de régression et les taux de décroissance associés aux trois massifs 
Tableau III.

Droites de régression et taux de décroissance des massifs prospectés.

\begin{tabular}{|l|c|c|}
\hline Massif forestier & $\begin{array}{c}\text { Équation } \\
\text { de régression }\end{array}$ & $\begin{array}{c}\text { Taux de } \\
\text { décroissance }\end{array}$ \\
\hline Senalba Gharbi & $y_{1}=-2,04 x_{1}+220$ & $11,3 \%$ \\
\hline Senalba Chergui & $y_{2}=-2,67 x_{2}+195$ & $12,4 \%$ \\
\hline Sehary Guebli & $y_{3}=-3,41 x_{3}+300$ & $7,9 \%$ \\
\hline
\end{tabular}

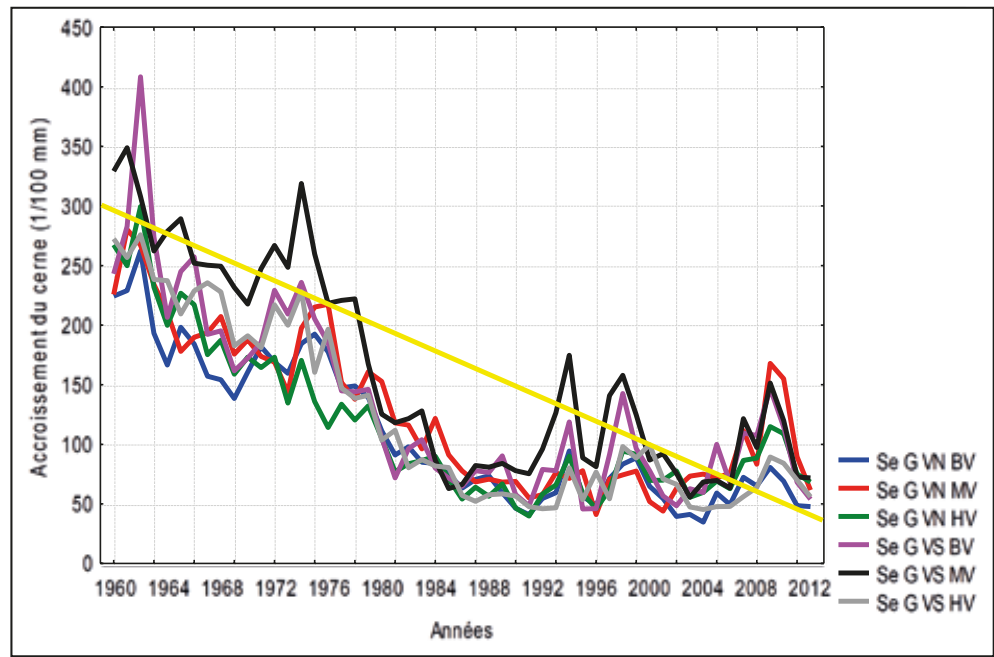

Figure 4.

Séries chronologiques, massif de Sehary Guebli.

Se G : Sehary Guebli ; VN : versant nord ; VS : versant sud ;

$\mathrm{BV}$ : bas versant ; MV : mi-versant ; HV : haut versant.

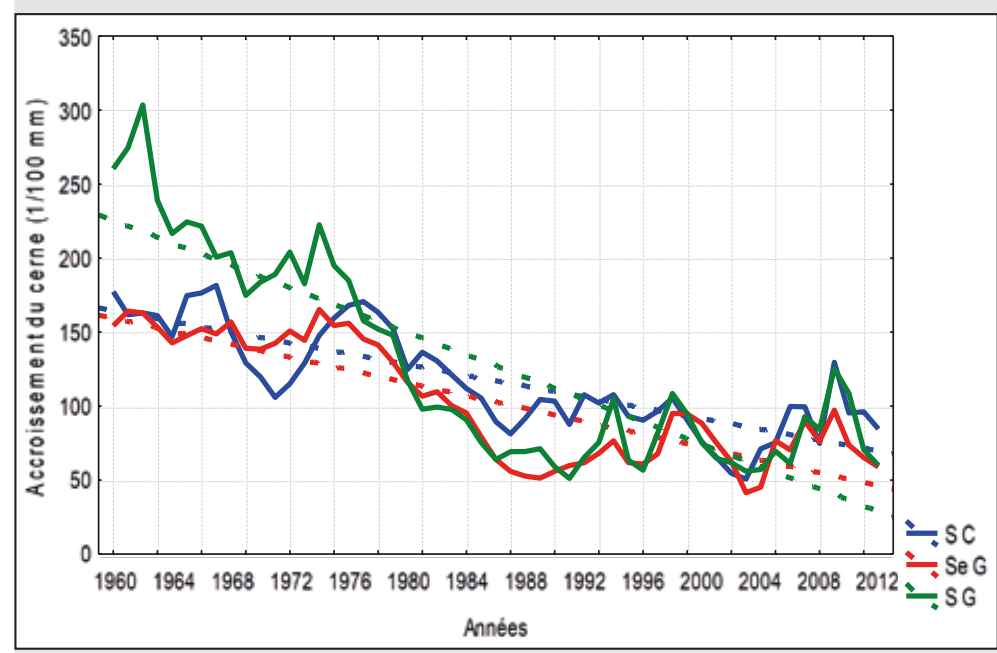

Figure 5.

Tendance globale sur les trois massifs.

S C : Senalba Chergui ; Se G : Sehary Guebli ;

$\mathrm{S} \mathrm{G}:$ Senalba Gharbi.
Trois explications (conjuguées ou séparées) peuvent être envisagées suite à cette diminution d'épaisseur des cernes : - La croissance radiale du pin d'Alep a baissé significativement pour la période 1960-2001. Cette diminution de productivité est attribuée à une diminution des précipitations et à une augmentation des températures provoquant une augmentation du stress hydrique (El Khorchani, 2006). À l'échelle régionale, la diminution de la croissance du pin d'Alep est de $22 \%$ en bioclimat aride, de $17 \%$ en bioclimat semi-aride et de $3 \%$ en bioclimat humide. À l'échelle locale, les réponses sont différentes selon les sites.

Ces résultats sont en accord avec ceux obtenus pour notre région d'étude avec un taux de décroissance de l'épaisseur des cernes compris entre $7,9 \%$ et $12,4 \%$ sur la période 1960-2012.

Cette décroissance est estimée en utilisant la décroissance moyenne entre les cinq décennies consécutives : 1960-1969, 1970-1979, 1980-1989, 1990-1999 et 2000-2009.

L’analyse des données climatiques de la région d'étude sur la période 1984-2013 (figure 6) met en évidence une tendance à l'aridité avec des températures en hausse et des précipitations en baisse pour la période trentenaire 1984-2013 dans la région d'étude. On enregistre une diminution générale des précipitations (estimée à - 18,3\% entre les deux décennies consécutives 1990-1999 et 2000-2009) et une augmentation générale des températures (estimée à $+2,5 \%$ entre les deux mêmes décennies consécutives).

- Dans une étude dendroécologique du pin d’Alep dans une région semi-aride de l'Atlas saharien algérien sur une période de 75 ans (1914-1988), Safar (1994) a distingué deux phases : une première phase de 1914 à 1950 et une seconde de 1951 à 1988 avec un accroissement des cernes qui a chuté considérablement entre les deux phases. Il observe une rupture de la croissance radiale du pin d'Alep entre ces deux phases du fait des changements climatiques. - La décroissance biologique normale des largeurs de cernes est en partie liée au vieillissement de l'arbre et à l'augmentation du diamètre du tronc. En effet, l'âge de formation d'un cerne affecte directement sa largeur. Les arbres jeunes présentent des accroissements de cernes plus marqués que les arbres âgés. En vieillissant, l'accroissement diminue progressivement jusqu'à la mort de l'arbre (Latte et al., 2012). Il faut rappeler que les arbres étudiés sont en phase de sénescence avec un âge moyen de 100 ans.

\section{Années caractéristiques de la chronologie}

Sur la période 1960-2012 (soit 53 années), le pin d'Alep de la région de Senalba Gharbi, Chergui et Sehary Guebli présente quatre années caractéristiques (1967, 1974, 1980 et 2009), soit un taux de $7,5 \%$. Ces années caractéristiques correspondent aux dates pour lesquelles au moins $75 \%$ des arbres du peuplement présentent une variation relative de 


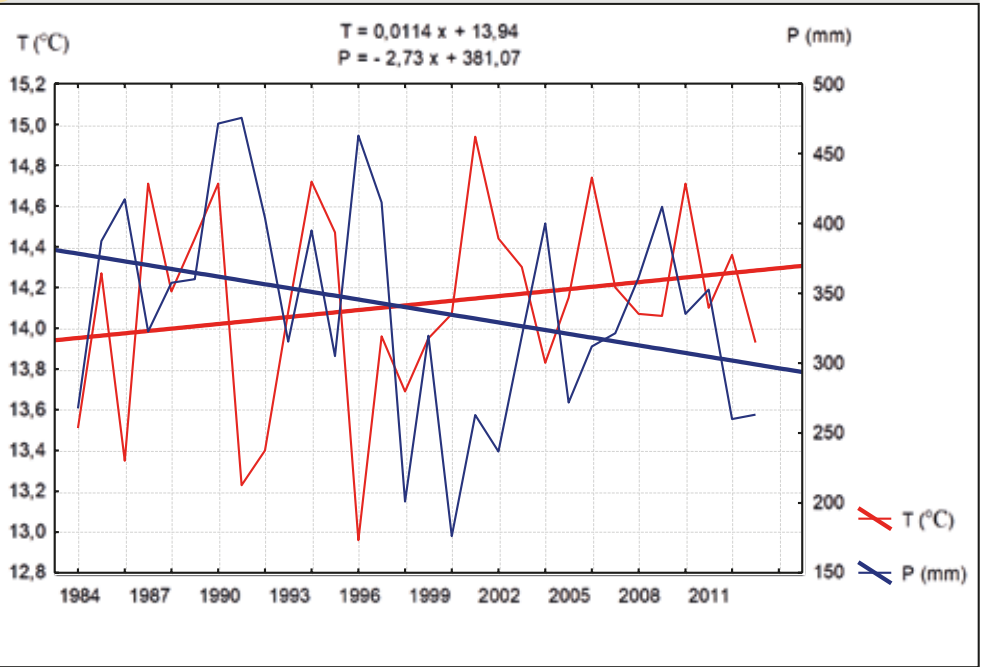

Figure 6.

Tendance climatique de la région d'étude sur la période 1984-2013.

croissance d'au moins 10 \% par rapport à l'année précédente. Elles se répartissent équitablement à raison d'une année caractéristique par décennie sauf pour la décennie 1990-1999. L'analyse des cernes montre qu'au cours de la décennie 2000-2009, jugée comme la plus chaude, sept années sur dix correspondent à un cerne mince. Un cerne est mince quand sa largeur est inférieure à celle du cerne moyen. L'année 2009, correspondant à un cerne large, est considérée comme la plus arrosée de la décennie 2000-2009.

\section{Quel avenir pour le pin d'Alep dans la région d'étude ?}

Il est admis que les changements climatiques, qui se traduisent par des sécheresses répétées, ont fortement affecté l'état sanitaire des peuplements forestiers (Sarris et al., 2011 ; Girard et al., 2012 ; Sanchez-Salguero et al., 2012). Dans les écosystèmes méditerranéens où l'eau est le principal facteur limitant, le phénomène de dépérissement est surtout ressenti dans les régions arides où les espèces forestières sont à la limite de leur aire de distribution (Linares et al., 2009 ; Allen et al., 2010 ; Babst et al., 2013).

Ces changements ont mis clairement le pin d'Alep en difficulté depuis quelques dizaines d'années dans la région semi-aride algérienne (notamment au niveau de Senalba Chergui, Senalba Gharbi et Sehary Guebli). Dans une étude sur l'état sanitaire des peuplements de pin d'Alep dans la même région de Senalba, Guit et al. (2016) ont conclu que les individus situés sur le versant sud souffrent davantage du phénomène de réchauffement climatique car ils se trouvent à la limite de leur aire écologique. Des dépérissements et réductions de productivité de l'espèce ont été observés également au sud de la Tunisie, en lien avec des séries d'années sèches et très chaudes (El Khorchani, 2006).

À l'issue de cette étude dendroécologique, l'état actuel des pinèdes de la zone d'étude appelle à des actions de réhabilitation afin de sauvegarder la biodiversité mena- cée par cette dégradation. Guit (2015) émet ainsi plusieurs recommandations à destination des décideurs du domaine forestier :

- Mettre en place des stations permanentes dans tout le domaine forestier afin de suivre simultanément les modifications de la croissance et de la santé des arbres ; sur le plan écologique, des suivis complémentaires sont nécessaires sur le cortège floristique des espèces arbustives et herbacées.

- Procéder à des traitements sylvicoles (coupes de régénération dans les peuplements sénescents, coupes d'assainissement pour éliminer les sujets dépéris).

- Conduire des programmes de reboisements afin de régénérer le patrimoine forestier. La reforestation doit se faire préférentiellement avec des semences de la même région, en considérant la variation biogéographique comme critère d'échantillonnage et de stratégie de collecte des graines.

- Mettre en défens les plantations en interdisant l'accès des jeunes semis au cheptel tout en accentuant les surveillances en mettant en place un gardiennage strict.

\section{Conclusion}

Dans la région semi-aride centre (Senalba et Sehary Guebli), l'exposition, l'altitude et la position topographique s'avèrent déterminantes pour la croissance radiale du pin d'Alep, Pinus halepensis Mill. L'analyse des séries chronologiques effectuée sur une période de 53 ans (de 1960 à 2012) a mis en évidence une diminution des accroissements des cernes du pin d'Alep dans la région d'étude, attribuée à une diminution des précipitations et une augmentation de la température, engendrant ainsi un stress hydrique accru.

Ces résultats permettent de prédire le comportement du pin d'Alep vis-à-vis du climat futur, sachant que le changement climatique aura plus d'impact sur ces peuplements situés dans la partie la plus méridionale de l'aire de répartition de l'espèce.

Les informations et données obtenues dans le cadre de cette étude pourront servir de base à la mise en place d'un outil de gestion forestière pour prévenir le phénomène de dépérissement des pins dans la région d'étude. Les futures recherches à conduire devraient également inclure des études écophysiologiques et entomologiques pour mieux évaluer l'état sanitaire des peuplements de pin d'Alep.

\section{Remerciements}

Le présent travail a pu être effectué grâce à l'aide financière du ministère algérien de l'Enseignement supérieur et de la Recherche scientifique (projet CNEPRU $n^{\circ}$ D04N01UN170120200003). Nous remercions vivement $M$. Frédéric Guibal, chercheur à l'Institut méditerranéen de biodiversité et d'écologie marine et continentale (Aix-enProvence, France), pour son accueil chaleureux au sein de son laboratoire de dendrochronologie. 


\section{Références}

Allen C. D., Macalady A. K., Chenchouni H., Bachelet D., Mc Dowell N., Vennetier M., et al., 2010. A global overview of drought and heat-induced tree mortality reveals emerging climate change risks for forests. Forest Ecology and Management, 259 (4): 660-684. https://doi.org/10.1016/i.foreco.2009.09.001

Babst F., Poulter B., Trouet V., Tan K., Neuwirth B., Wilson R., et al., 2013. Site and species specific responses of forest growth to climate across the European continent. Global Ecology and Biogeography, 22: 706-717. https://doi. org/10.1111/geb.12023

Bentouati A., Oudjehih B., Alatou D., 2005. Croissance en hauteur dominante et classes de fertilité du pin d'Alep (Pinus halepensis Mill.) dans le massif de Ouled Yakoub et des Beni Oudjana (Khenchela-Aures). Sciences \& Technologie, 23 : 57-62. http://revue.umc.edu.dz/index.php/c/\%20article/view/1379

Brochiero F., Chandioux O., Ripert C., Vennetier M., 1999. Autécologie et croissance du pin d'Alep en Provence calcaire. Forêt Méditerranéenne, 20 : 83-94. http://hdl.handle.net/2042/40913

Carmel Y., Kadmon R., 1999. Effects of grazing and topography on long-term vegetation changes in a Mediterranean ecosystem in Israel. Plant Ecology, 145: 243-254. https://doi.org/10.1023/A:1009872306093

De Dios V. R., Fischer C., Colinas C., 2007. Climate change effects on Mediterranean forest and preventive measures. New Forests, 33: 29-40. https://doi. org/10.1007/s11056-006-9011-x

De Luis M., Čufar K., Di Filippo A., Novak K., Papadopoulos A., Piovesan G., et al., 2013. Plasticity in Dendroclimatic Response across the Distribution Range of Aleppo Pine (Pinus halepensis). PLOS One, 9 (1). https://doi.org/10.1371/ journal.pone.0083550

D’Orangeville L., Duchesne L., Houle D., Kneeshaw D., Côte B., Pederson N., 2016. Northeastern North America as a potential refugium for boreal forests in a warming climate. Science, 352: 1452-1455. https://doi.org/10.1126/ science.aaf4951

El Khorchani A., 2006. Impact des changements climatiques récents sur la croissance radiale de trois pins méditerranéens (pin d'Alep, pin pignon et pin maritime) en Tunisie. Marseille, France, IMEP, Aix-Marseille III, 170 p.

Fritts H. C., 2001. Tree Rings and Climate. Caldwell, NJ, USA, The Blackburn Press, 584 p. https:// doi.org/10.1002/iqs.796

Fritts H. C., Swetnam T. W., 1989. Dendroecology: A Tool for Evaluating Variations in Past and Present Forest Environments. Advances in Ecological Research, 19: 111-188. https://doi.org/10.1016/S0065-2504(08)60158-0

Girard F., Vennetier M., Guibal F., Corona C., Ouarmim S., Herrero A., 2012. Pinus halepensis Mill. crown development and fruiting declined with repeated drought in Mediterranean France. European Journal of Forest Research, 131: 919-931. https://doi.org/10.1007/s10342-011-0565-6

Girardin M. P., Bouriaud O., Hogg E. H., Kurz W., Zimmermann N. E., Metsaranta J. M., et al., 2016. No growth stimulation of Canada's boreal forest under half-century of combined warming and $\mathrm{CO}_{2}$ fertilization. Proceedings of the National Academy of Sciences, 113 (52): 8406-8414. www.pnas.org/cgi/ doi/10.1073/pnas.1610156113

Guit B., 2015. Croissance et état sanitaire des peuplements de pin d'Alep (Pinus halepensis Mill.) dans le massif forestier de Senalba (Région de Djelfa). Thèse de doctorat, ENSA, Alger, $107 \mathrm{p}$.

Guit B., Nedjimi B., Guibal F., Chakali G., 2015. Dendroécologie du pin d’Alep (Pinus halepensis Mill.) en fonction des paramètres stationnels dans le massif forestier de Senalba (Djelfa, Algérie). Revue Écologie (Terre et Vie), 70 : 32-43. http://hdl.handle.net/2042/56308

Guit B., Nedjimi B., Chakali G., Guibal F., 2016. État sanitaire des peuplements de pin d'Alep (Pinus halepensis Mill.) dans le massif forestier de Senalba (Djelfa, Algérie). Revue Écologie (Terre et Vie), $71: 156-167$. http:// hdl.handle.net/2042/59913

IPCC, 2007. Climate change: the physical science basis. Contribution of Working Group I to the Fourth Assessment Report of the Intergovernmental Panel on Climate Change. Solomon S., Qin D., Manning M., Chen Z., Marquis M., Averty K. B., et al. (eds). Cambridge University Press, Cambridge, United Kingdom/New York, NY, USA, 996 p. www.cambridge.org/9780521880091

Kaabèche M., 1996. Les relations climat-végétation dans le bassin du Hodna (Algérie). Acta Botanica Gallica, 143 (1) : 85-94. https://doi.org/10.1080/12 538078.1996.10515321

Kadik B., 1987. Contribution à l'étude du pin d'Alep (Pinus halepensis Mill.) en Algérie. Écologie, dendrométrie, morphologie. Alger, Algérie, Office des publications universitaires, $580 \mathrm{p}$.

Kadik-Achoubi L., 2005. Étude phytosociologique et phytoécologique des formations à pin d'Alep (Pinus halepensis Mill.) de l'étage bioclimatique semiaride algérien. Thèse de doctorat, USTHB, Alger, $341 \mathrm{p}$.

Latte N., Debruxelles J., Sohier C., Degré A., Claessens H., 2012. La dendroécologie : Un outil pour affiner nos connaissances sur l'autécologie des essences forestières. Forêt Wallonne, 116 : 3-17. http://hdl.handle.net/2268/113099
Bois et Forêts des Tropiques - ISSN : L-0006-579X

Lebourgeois F., Mérian P., 2012. Principes et méthodes de la dendrochronologie. LERFOB, AgroParisTech, Centre de Nancy, 85 p. http://hal.archives-ouvertes.fr/cel-01627048

Linares J. C., Camarero J. J., Carreira J. A., 2009. Interacting effects of changes in climate and forest cover on mortality and growth of the southernmost European fir forests. Global Ecology and Biogeography, 18: 485-497. https://doi. org/10.1111/j.1466-8238.2009.00465.X

Lindner M., Maroschek M., Netherer S., Kremer A., Barbati A., Garcia-Gonzalo J., et al., 2010. Climate change impacts, adaptive capacity, and vulnerability of European forest ecosystems. Forest Ecology and Management, 259 (4): 698-709. https://doi.org/10.1016/j.foreco.2009.09.023

Ne'eman G., Trabaud L. (eds), 2000. Ecology, Biogeography and Management of Pinus halepensis and $P$. brutia Forest Ecosystems in the Mediterranean Basin. Leiden, the Netherlands, Backhuys Publishers, 407 p. https://doi org/10.1046/j.0022-0477.2001.00591.x

Osem Y., Zangy E., Bney-Moshe E., Moshe Y., Karni N., Nisan Y., 2009. The potential of transforming simple structured pine plantations into mixed Mediterranean forests through natural regeneration along a rainfall gradient. Forest Ecology and Management, 259 (1): 14-23. https://doi.org/10.1016/i. foreco.2009.09.034

Ruiz-Labourdette D., Fe Schmitz M., Pineda F. D., 2013. Changes in tree species composition in Mediterranean mountains under climate change: Indicators for conservation planning. Ecological Indicators, 24: 310-323. https:// doi.org/10.1016/j.ecolind.2012.06.021

Safar W., 1994. Contribution à l'étude dendroécologique du pin d’Alep (Pinus halepensis Mill.) dans une zone semi-aride d'Algérie : l'Atlas saharien (Ouled Naïl, Aurès, Hodna). Thèse de doctorat, Faculté Saint-Jérôme, Aix-Marseille III, France, 215 p

Sanchez-Salguero R., Navarro-Cerrillo R. M., Camarero J. J., Fernandez-Cancio A., 2012. Selective drought-induced decline of pine species in southeastern Spain. Climatic Change, 113: 767-785. https://doi.org/10.1007/s10584-011-0372-6 Sarris D., Christodoulakis D., Körner C., 2011. Impact of recent climatic change on growth of low elevation eastern Mediterranean forest trees. Climatic Change, 106: 203-223. https://doi.org/10.1007/s10584-010-9901-y

Schiller G., 1972. Ecological factors affecting the growth of Aleppo pine in the southern Judean hills. Leaflet, Agricultural Research Organisation. Llanot, 44 20 p. (In Hebrew)

\begin{tabular}{|c|c|}
\hline Rôle du contributeur & Noms des auteurs \\
\hline Conceptualisation & B. Guit, B. Nedjimi \\
\hline Gestion des données & B. Guit \\
\hline Analyse formelle & B. Guit, B. Nedjimi \\
\hline $\begin{array}{l}\text { Acquisition } \\
\text { du financement }\end{array}$ & B. Nedjimi \\
\hline $\begin{array}{l}\text { Enquête } \\
\text { et investigation }\end{array}$ & B. Nedjimi, B. Guit \\
\hline Méthodologie & B. Guit, B. Nedjimi \\
\hline Gestion de projet & B. Nedjimi \\
\hline Ressources & B. Nedjimi, B. Guit \\
\hline Logiciels & B. Guit, B. Nedjimi \\
\hline Supervision & B. Nedjimi \\
\hline Validation & B. Guit, B. Nedjimi \\
\hline Visualisation & B. Guit, B. Nedjimi \\
\hline $\begin{array}{l}\text { Écriture - Préparation } \\
\text { de l'ébauche originale }\end{array}$ & B. Nedjimi \\
\hline $\begin{array}{l}\text { Écriture - Révision } \\
\text { et édition }\end{array}$ & B. Guit, B. Nedjimi \\
\hline
\end{tabular}

Bois et Forêts des Tropiques - Revue scientifique du Cirad

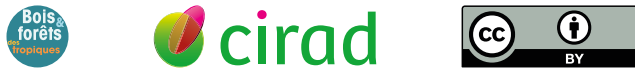

Cirad - Campus international de Baillarguet, 34398 Montpellier

Cedex 5, France - Contact : bft@cirad.fr - ISSN : L-0006-579X 